\section{Incontinência urinária em mulheres que buscam exame preventivo de câncer de colo uterino: fatores sociodemográficos e comportamentais}

\author{
Urinary incontinence in women undergoing Pap \\ smear test: socio-demographic and behavioral \\ factors \\ Incontinencia urinaria en mujeres que solicitan un \\ examen preventivo de cáncer de cuello uterino: \\ factores socio-demográficos y de comportamiento
}

\author{
${ }_{1}$ Universidade do Estado \\ de Santa Catarina, \\ Florianópolis, Brasil. \\ 2 Universidade Federal \\ de Santa Catarina, \\ Florianópolis, Brasil. \\ Correspondência \\ C. Sacomori \\ Universidade do Estado de \\ Santa Catarina. \\ Rua Pascoal Simone 358 \\ Florianópolis, SC 88080-350, \\ Brasil. \\ csacomori@yahoo.com.br
}

\begin{abstract}
This cross-sectional study aimed to investigate the association between socio-demographic and behavioral factors and the presence of selfreported urinary incontinence in 784 women undergoing cervical cancer screening in Greater Florianópolis, Santa Catarina State, Brazil. Socio-demographic data, health status, physical activity, constipation, and body mass index were obtained, and the International Consultation on Incontinence Questionnaire-SF was used. Prevalence of urinary incontinence was $30.7 \%$ (16.5\% reported leakage once a week or less and $23.8 \%$ losses in small volumes). Poisson univariate regression showed the following factors associated with urinary incontinence: lower education, lower income, non-white skin color, overweight, worse health status, constipation, and older age. After adjustment, according to a hierarchical model, schooling, ethnicity, health status, and age remained significantly associated. The high prevalence of urinary incontinence in women seeking Pap smear tests justifies preventive approaches in these areas of intervention.
\end{abstract}

Urinary Incontinence; Women's Health; Risk Factors; Mass Screening; Motor Activity
Cinara Sacomori 1,2

Nubia Berenice Negri 1

Fernando Luiz Cardoso 1

\section{Resumo}

Este estudo transversal objetivou investigar a associação entre, de um lado, fatores sociodemográficos e comportamentais e, de outro, a presença de incontinência urinária referida em 784 mulheres que buscam exame preventivo de câncer de colo uterino na Grande Florianópolis, Santa Catarina, Brasil. Foram obtidos dados sociodemográficos, estado de saúde, atividade física, constipação e índice de massa corporal, sendo utilizado o International Consultation on Incontinence Questionnaire-SF. A prevalência de incontinência urinária foi de 30,7\% (16,5\% perdiam urina uma vez por semana ou menos e 23,8\% em pequena quantidade). Na regressão de Poisson bruta, estiveram associados à incontinência urinária os seguintes fatores: baixa escolaridade, renda por pessoa da família até um salário mínimo, etnia não caucasiana, excesso de peso corporal, pior autoavaliação do estado de saúde, constipação e idade. Após ajuste, seguindo modelo hierarquizado, permaneceram associados: escolaridade, etnia, estado de saúde e idade. A alta prevalência de incontinência urinária em mulheres que buscam exame de rastreamento do câncer de colo uterino justifica abordagens preventivas nesses espaços de atuação.

Incontinência Urinária; Saúde da Mulher; Fatores de Risco; Programas de Rastreamento; Atividade Motora 


\section{Introdução}

De acordo com a Sociedade Internacional de Continência, a incontinência urinária é considerada a queixa de qualquer perda involuntária de urina 1. A sua prevalência em mulheres pode variar de $10 \%$ a $55 \%$ em todo o mundo, dependendo da população estudada e do critério empregado para o diagnóstico, o que a torna um problema de ordem de saúde pública que merece maior atenção ${ }^{1}$. A maioria das mulheres ignora os sintomas de incontinência urinária no início, e o avanço destes acaba impactando a qualidade de vida, com prejuízos emocionais, sociais e físicos 2 .

Estudos de base populacional têm identificado alguns fatores associados à incontinência urinária: sociodemográficos (idade $3,4,5$, residência em cidades em relação ao campo ${ }^{6}$, casamento mais jovem 3 , suporte social fraco 7 ), biológicos (menopausa, fatores hereditários 8), obstétricos (número de partos e partos vaginais $5,6,8$ ) e comportamentais (presença de diabetes 6 , índice de massa corporal elevado 5,7,9 e estado de saúde precário 7). No contexto de Brasil, são poucos os estudos epidemiológicos sobre os fatores associados à incontinência urinária. Enquanto um inquérito domiciliar 10 não identificou associação com aspectos socioeconômicos e reprodutivos em mulheres climatéricas, um estudo caso-controle 11, também realizado no Estado de São Paulo, identificou associação com variáveis obstétricas.

Dos fatores supracitados, os comportamentais são passíveis de modificação; por isso, fazse necessária uma abertura de espaços em que esses aspectos sejam investigados e minimizados. Dentre eles, na atenção à saúde da mulher, destacam-se a estrutura da atenção primária e a Rede Feminina de Combate ao Câncer (ONG), as quais oferecem o serviço de exames de rastreamento de câncer de colo uterino. Estudos epidemiológicos têm demonstrado que a cobertura desses exames é satisfatória no Brasil 12,13, sendo esse ambiente propício para detecção e cuidado de outros problemas de saúde que prejudicam a qualidade de vida da mulher, como é o caso da incontinência urinária.

Dado o exposto, o objetivo do presente estudo foi investigar a associação entre fatores sociodemográficos e comportamentais, de um lado, e o autorrelato de incontinência urinária em mulheres que buscam por exames de rastreamento do câncer de colo uterino, de outro. A hipótese deste estudo é que os fatores comportamentais (sedentarismo, constipação, obesidade, pior estado de saúde) e sociodemográficos (maior idade, baixa escolaridade, menor renda, etnia caucasiana) estão associados a uma maior probabilidade de desenvolvimento de incontinência urinária.

\section{Materiais e métodos}

Trata-se de uma pesquisa observacional que utilizou um desenho de estudo transversal.

\section{Participantes}

Participaram do estudo 784 mulheres que frequentaram a Rede Feminina de Combate ao Câncer no Município de Florianópolis, Santa Catarina, Brasil, com idade entre 18 e 89 anos. A referida instituição oferece qualidade e tradição no serviço prestado, atendendo anualmente cerca de 4.200 mulheres de todas as classes sociais, sendo a amostra deste estudo equivalente a aproximadamente $18 \%$ dessa população.

Todas as mulheres realizaram o exame preventivo de câncer de colo de útero e aceitaram participar do estudo por meio da assinatura do termo de consentimento livre e esclarecido do projeto de pesquisa aprovado junto ao Comitê de Ética em Pesquisa da Universidade do Estado de Santa Catarina (UDESC; no 15/2011). Foram avaliadas todas aquelas que compareciam à instituição, sem horário marcado, às terças, quintas e sextas-feiras, no período vespertino, entre os meses de setembro de 2011 a junho de 2012. As avaliações ocorreram nesses dias específicos em virtude da disponibilidade de sala na instituição. Os critérios de exclusão do estudo incluíram gestação, incapacidade física, déficit cognitivo e idade inferior a 18 anos.

\section{Instrumentos}

Todas as mulheres participantes desta pesquisa responderam a um questionário contendo perguntas relacionadas à prática de atividade física no deslocamento, em casa, no trabalho e no lazer. As questões eram idênticas às utilizadas no estudo do Instituto Brasileiro de Geografia e Estatística (IBGE) quando da realização da Pesquisa Nacional por Amostra de Domicílios (http:// www.ibge.gov.br/home/estatistica/populacao/ trabalhoerendimento/pnad2009, acessado em 05/Out/2011). Foram consideradas como mulheres ativas no lazer as que disseram que "nos três últimos meses praticaram algum tipo de exercício físico ou esporte". As ativas no deslocamento eram as mulheres que "costumavam ir a pé ou de bicicleta de casa para o trabalho". Ativas no trabalho corresponderam àquelas que "no trabalho andavam a maior parte do tempo, carregavam peso ou faziam outra atividade que requeria es- 
forço físico intenso". Foram consideradas ativas em casa as que afirmaram que "costumavam fazer a faxina (limpeza pesada) no domicílio".

Além disso, as participantes responderam sobre dados sociodemográficos, autoavaliação do estado de saúde, constipação e frequência de perdas urinárias. Os indicadores do índice de massa corporal (IMC) foram obtidos com balança digital para massa corporal e fita métrica fixada à parede para altura. A constipação foi avaliada utilizando-se os critérios de Roma III, constituído por critérios objetivos (frequência de evacuações, necessidade de manobras manuais para defecar) e subjetivos (esforço ao evacuar, evacuação incompleta, sensação de obstrução, fezes duras) ${ }^{14}$. No presente estudo, foram consideradas constipadas as mulheres que apresentavam pelo menos três desses critérios.

Para avaliação de perdas urinárias, aplicouse o Questionário Internacional de Incontinência [International Consultation on Incontinence Questionnaire-Short Form (ICIQ-SF)], que avalia o impacto da incontinência urinária sobre qualidade de vida e também qualifica a perda urinária de pacientes de ambos os sexos. O ICIQ-SF foi traduzido para a língua portuguesa e validado por Tamanini et al. 15. O instrumento é composto por quatro questões que avaliam a frequência, a gravidade e o impacto da incontinência urinária, além de um conjunto de oito itens relacionados às causas ou situações de incontinência urinária vivenciadas pelas pacientes 15 (Figura 1).

\section{Procedimentos}

Os dados foram coletados no momento em que as mulheres aguardavam na sala de espera para realização do exame de rastreamento do câncer de colo uterino. Elas foram convidadas a participar do estudo antes de realizarem o exame, sendo explicados o objetivo da pesquisa e o sigilo da identificação.

A coleta foi realizada por uma fisioterapeuta e duas alunas do curso de Fisioterapia, previamente treinadas, e ocorreu em privacidade. Pouquíssimas mulheres negaram-se a participar, pois a sua participação não implicava um gasto maior de tempo. Primeiramente, foram coletados os dados de caracterização da amostra; em seguida, aplicaram-se os demais instrumentos de pesquisa.

\section{Análise dos dados}

Os dados foram tabulados no programa estatístico SPSS versão 18.0 (SPSS Inc., Chicago, Estados Unidos) e analisados com recursos da estatística descritiva (frequência absoluta e relativa, média e desvio-padrão) e inferencial. A fim de analisar as associações entre a variável dependente (incontinência urinária) e as variáveis independentes (sociodemográficas e comportamentais), procedeu-se à análise de regressão de Poisson com variância robusta para obtenção de estimativas brutas e ajustadas das razões de prevalência, considerando $\mathrm{p}<0,05$. Para as análises ajustadas, adotou-se a mesma metodologia de Mascarenhas et al. 16, obedecendo à ordem de um modelo hierárquico, para determinação dos desfechos em que foram incluídas as variáveis que apresentaram $\mathrm{p}<0,25$ nas análises brutas 17 . As variáveis do nível 1 (sociodemográficas) foram ajustadas entre si, sendo mantidas no modelo aquelas que obtiveram $\mathrm{p}<0,25$, para ajuste das variáveis no nível subsequente. A seguir, foram incluídas as variáveis do nível 2 (comportamentais) que obtiveram $\mathrm{p}<0,25$ na análise bruta juntamente com as do nível 1 , para o ajustamento das variáveis.

Foram consideradas incontinentes as mulheres que referiam perda de urina nas frequências de uma vez por semana ou menos, duas vezes por semana ou menos, uma vez ao dia, diversas vezes ao dia ou o tempo todo.

Para categorização da idade em faixas etárias, considerando que este estudo não controlou se as mulheres estavam ou não na menopausa, optou-se por três categorias: 0 (até 44 anos), 1 (de 45 a 55 anos) e 2 (com 56 anos ou mais). O critério para tal classificação teve por base o estudo de Pedro et al. ${ }^{18}$, cujos autores citam que a média de idade da menopausa em mulheres brasileiras foi de 51 anos, sendo mais frequente sua ocorrência entre 45 e 55 anos.

\section{Resultados}

A média de idade das mulheres foi de 39,62 anos (DP $=13,29$ ), e a média da renda por pessoa da família correspondia a 1,25 salário mínimo $(\mathrm{DP}=$ 0,91 ). A maioria era de etnia caucasiana, havia cursado nível de escolaridade fundamental e médio, tinha parceiros sexuais e considerava ter um bom estado de saúde. Em relação aos domínios de atividade física, predominaram as mulheres ativas em atividades domésticas.

A prevalência de incontinência urinária referida foi de $30,7 \%(n=241)$. Quanto à perda de urina, $16,5 \%$ das mulheres $(\mathrm{n}=129)$ referiram perdê-la uma vez por semana ou menos; $5,6 \%$ $(\mathrm{n}=44)$, duas vezes por semana ou menos; $3,8 \%$ $(\mathrm{n}=30)$, uma vez ao dia; $4,5 \%(\mathrm{n}=35)$, diversas vezes ao dia; e $0,4 \%(n=3)$ disse perder urina o tempo todo. No que se refere à intensidade das perdas, a maioria das participantes mencionou 


\begin{tabular}{|c|c|c|}
\hline \multicolumn{3}{|l|}{ 3. Com que frequência você perde urina? } \\
\hline ( ) Nunca- 0 & ( ) Uma vez por semana ou menos - 1 & ( ) Duas ou três vezes por semana -2 \\
\hline ( ) Uma vez ao dia - 3 & ( ) Diversas vezes ao dia - 4 & ( ) O tempo todo -5 \\
\hline \multicolumn{3}{|c|}{ 4. Qual é a quantidade de urina que você pensa perder? } \\
\hline ( ) Nenhuma - 0 & \multicolumn{2}{|l|}{ ( ) Uma pequena quantidade -2} \\
\hline ( ) Uma moderada quantidade -4 & \multicolumn{2}{|l|}{ ( ) Uma grande quantidade -6} \\
\hline \multicolumn{3}{|c|}{ 5. Em geral, quanto perder urina interfere em sua vida diária? } \\
\hline \multicolumn{3}{|l|}{$\begin{array}{lllllllllll}0 & 1 & 2 & 3 & 4 & 5 & 6 & 7 & 8 & 9 & 10\end{array}$} \\
\hline \multicolumn{3}{|c|}{ Não interfere $\quad$ Interfere muito } \\
\hline \multicolumn{3}{|c|}{ ICIQ Escore: soma dos resultados $3+4+5=$} \\
\hline \multicolumn{3}{|l|}{ Quando você perde urina? } \\
\hline \multicolumn{2}{|l|}{ ( ) Nunca perco } & ( ) Perco antes de chegar ao banheiro \\
\hline \multicolumn{2}{|l|}{ ( ) Perco quando tusso ou espirro } & ( ) Perco quando estou dormindo \\
\hline ( ) Perco quando estou fazendo atividades físicas & & ( ) Perco quando terminei de urinar e estou me vestindo \\
\hline ( ) Perco sem razão óbvia & & ( ) Perco o tempo todo \\
\hline
\end{tabular}

ser em pequena quantidade (186 mulheres; 23,8\%), 32 (4,1\%) afirmaram perder quantidade moderada e $21(2,7 \%)$ disseram perder grande quantidade de urina.

Em relação às situações de perda de urina, 93 mulheres $(11,9 \%)$ perdiam antes de chegar ao banheiro; 206 (26,3\%), durante tosse ou espirro; $14(1,8 \%)$, enquanto dormiam; $70(9 \%)$, praticando atividades físicas; 52 (6,6\%), quando estavam terminando de urinar e começavam a se vestir; e 31 (4\%), sem razão óbvia.

Os fatores associados à incontinência urinária referida estão apresentados na Tabela 1. Nas análises brutas, encontrou-se associação entre incontinência urinária e algumas variáveis sociodemográficas e comportamentais. Dentre os fatores sociodemográficos, estiveram associados à incontinência urinária referida: faixa etária, renda por pessoa na família, escolaridade e etnia. Em relação à faixa etária, a maior proporção de mulheres com incontinência foi observada entre aquelas com idade maior ou igual a 56 anos (51,4\%) e com 45 a 55 anos (36,9\%). A maior prevalência de incontinência urinária referida ocorreu entre as mulheres cuja renda por pessoa da família era de até um salário mínimo, com escolaridade baixa (ensino fundamental completo/incompleto) e de etnia não caucasiana. Dos fatores comportamentais investigados, ainda na análise bruta, estiveram associados à incontinência urinária referida: presença de sobrepeso/obesidade, autoavaliação do estado de saúde como regular/ruim/muito ruim e presença de constipação intestinal.

Após os ajustes intra e interníveis (Tabela 1), de acordo com o modelo hierárquico, as variáveis da atividade física não permaneceram no modelo por não atenderem ao critério de significância $(\mathrm{p}<0,25)$. Os resultados da análise ajustada evidenciaram que as seguintes variáveis estavam associadas à incontinência urinária autorreferida: faixa etária, escolaridade, etnia e estado de saúde.

\section{Discussão}

Por meio de autorrelato feito em questionário validado, foi avaliada a prevalência de incontinência urinária na população de mulheres que buscam por exames preventivos de câncer de colo do útero. Embora estudos de base populacional tenham demonstrado que a proporção de mulheres que realizam esse exame é satisfatória 12,13 , esta pesquisa limita-se por avaliar a incontinência urinária referida em um único serviço público de saúde, o que não reflete a população geral brasileira. 
Tabela 1

Fatores sociodemográficos e comportamentais associados à incontinência urinária.

\begin{tabular}{|c|c|c|c|c|c|c|}
\hline \multirow[t]{2}{*}{ Variáveis } & \multicolumn{2}{|c|}{$\begin{array}{l}\text { Continentes } \\
(n=543)\end{array}$} & \multicolumn{2}{|c|}{$\begin{array}{l}\text { Incontinentes } \\
(n=241)\end{array}$} & \multirow{2}{*}{$\begin{array}{l}\text { Análise bruta } \\
\text { RP (IC) }\end{array}$} & \multirow{2}{*}{$\begin{array}{c}\text { Análise ajustada } \\
\text { RP (IC) }\end{array}$} \\
\hline & $\mathrm{n}$ & $\%$ * & $\mathrm{n}$ & $\%$ * & & \\
\hline \multicolumn{7}{|l|}{ Nível 1} \\
\hline \multicolumn{7}{|l|}{ Sociodemográficas } \\
\hline \multicolumn{7}{|l|}{ Faixa etária (anos) } \\
\hline Até 44 & 376 & 76,1 & 118 & 23,9 & 1,00 & 1,00 \\
\hline $45-55$ & 106 & 63,1 & 62 & 36,9 & $1,54(1,20-1,99)$ ** & $1,35(1,03-1,76)$ ** \\
\hline 56 ou mais & 52 & 48,6 & 55 & 51,4 & $2,15(1,69-2,74) * *$ & $1,88(1,44-2,44) * *$ \\
\hline \multicolumn{7}{|l|}{ Escolaridade } \\
\hline Ensino Fundamental completo/incompleto & 195 & 58,0 & 141 & 42,0 & $1,90(1,54-2,36)$ ** & $1,59(1,24-2,03) * *$ \\
\hline Ensino Médio/Superior completo/incompleto & 343 & 78,0 & 97 & 22,0 & 1,00 & 1,00 \\
\hline \multicolumn{7}{|l|}{ Renda família/pessoa (salário mínimo) } \\
\hline Até 1 & 313 & 66,6 & 157 & 33,4 & $1,30(1,03-1,64) * *$ & $1,20(0,95-1,52)$ \\
\hline$>1$ & 220 & 74,3 & 76 & 25,7 & 1,00 & 1,00 \\
\hline \multicolumn{7}{|l|}{ Estado marital } \\
\hline Tem parceiro & 371 & 67,5 & 179 & 32,5 & $1,23(0,96-1,56)$ & $1,26(0,99-1,60)$ \\
\hline Não tem parceiro & 172 & 73,5 & 62 & 26,5 & 1,00 & 1,00 \\
\hline \multicolumn{7}{|l|}{ Etnia } \\
\hline Caucasiana & 437 & 70,9 & 179 & 29,1 & 1,00 & 1,00 \\
\hline Negra/Mulata/Amarela & 106 & 63,1 & 62 & 36,9 & $1,27(1,01-1,60) * *$ & $1,30(1,02-1,65)$ ** \\
\hline \multicolumn{7}{|l|}{ Nível 2} \\
\hline \multicolumn{7}{|l|}{ Comportamentais } \\
\hline \multicolumn{7}{|l|}{ Classificação no IMC } \\
\hline Abaixo do peso/Peso normal & 290 & 79,5 & 75 & 20,5 & 1,00 & 1,00 \\
\hline Sobrepeso/Obesidade & 225 & 61,1 & 143 & 38,9 & $1,89(1,49-2,40) * *$ & $1,26(0,96-1,65)$ \\
\hline \multicolumn{7}{|l|}{ Estado de saúde } \\
\hline Muito bom/Bom & 345 & 75,5 & 112 & 24,5 & 1,00 & 1,00 \\
\hline Regular/Ruim/Muito ruim & 197 & 61,2 & 125 & 38,8 & $1,58(1,28-1,96) * *$ & $1,35(1,04-1,76) * *$ \\
\hline \multicolumn{7}{|l|}{ Constipação } \\
\hline Sim & 87 & 58,8 & 61 & 41,2 & $1,31(1,04-1,66) * \star$ & $1,21(0,94-1,55)$ \\
\hline Não & 313 & 68,6 & 143 & 31,4 & 1,00 & 1,00 \\
\hline \multicolumn{7}{|l|}{ Atividade física no lazer } \\
\hline Sim & 262 & 70,2 & 111 & 29,8 & 1,00 & *** \\
\hline Não & 281 & 68,4 & 130 & 31,6 & $1,06(0,86-1,31)$ & \\
\hline \multicolumn{7}{|l|}{ Atividade física no trabalho } \\
\hline Sim & 245 & 69,4 & 108 & 30,6 & 1,00 & 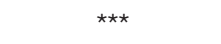 \\
\hline Não & 298 & 69,1 & 133 & 30,9 & $1(0,81-1,24)$ & \\
\hline \multicolumn{7}{|l|}{ Atividade física no deslocamento } \\
\hline Sim & 150 & 67,3 & 73 & 32,7 & 1,00 & 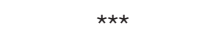 \\
\hline Não & 393 & 70,1 & 168 & 29,9 & $0,91(0,73-1,15)$ & \\
\hline \multicolumn{7}{|l|}{ Atividade física doméstica } \\
\hline Sim & 378 & 68,0 & 178 & 32,0 & 1,00 & 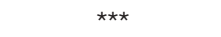 \\
\hline Não & 165 & 72,4 & 63 & 27,6 & $0,86(0,67-1,10)$ & \\
\hline
\end{tabular}

IC: intervalo de confiança; IMC: índice de massa corporal; RP: razão de prevalência.

* Foram utilizadas porcentagens válidas em virtude de alguns dados faltantes em algumas variáveis;

** Significativo para $\mathrm{p}<0,05$;

*** Variáveis não inseridas no modelo de Poisson múltiplo por não apresentarem $p<0,25$. 
A prevalência de incontinência urinária autorreferida foi de $30,7 \%$; enquanto $16,5 \%$ das mulheres referiram perdas uma vez por semana ou menos, $23,8 \%$ relataram perdas em pequena quantidade. Waetjen et al. 7 analisaram mulheres na meia-idade nos Estados Unidos, tendo verificado que $46,7 \%$ mencionavam alguma perda de urina e $15,3 \%$ a perdiam diversas vezes por semana. Estudo de base populacional na Noruega 4 identificou prevalência de $25 \%$ e, aproximadamente, $7 \%$ apresentavam incontinência moderada ou grave.

Uma metanálise 19 verificou taxa de prevalência que variava de $10 \%$ a $50 \%$ na população mundial, sendo o pico na meia-idade. Tal variação nas taxas de prevalência deve-se, principalmente, aos critérios do estudo, tais como instrumento de pesquisa utilizado, gravidade dos sintomas, idade abrangida e tipo de incontinência urinária investigado (esforço, urgência ou mista). Em suma, a prevalência de incontinência urinária referida em mulheres que buscam exames preventivos de câncer de colo uterino foi similar à de outros estudos epidemiológicos.

Com o objetivo de avaliar o papel dos fatores sociodemográficos no desenvolvimento de incontinência urinária, estudos prévios identificaram baixa prevalência dessa afecção em sociedades simples - tribos indígenas - no Brasil e nos Estados Unidos 20,21. Na tribo brasileira, os fatores de risco encontrados foram semelhantes aos das sociedades complexas: número de partos vaginais e idade. Estima-se que o estilo de vida desses grupos humanos e o uso da posição de cócoras possam prevenir algumas lesões no parto e, por consequência, evitar disfunções do assoalho pélvico 20.

Dentre os fatores sociodemográficos aqui investigados, estiveram associados à incontinência urinária autorreferida após análise ajustada: faixa etária, etnia e escolaridade. A renda por pessoa da família mostrou associação apenas na análise bruta. Um estudo sobre essa temática concluiu que o conhecimento que as mulheres tinham sobre a incontinência urinária estava associado a seu status socioeconômico, o qual era obtido conforme a ocupação do responsável pelo domicílio e o nível de escolaridade da mulher 22.

A maior parte dos estudos sobre a incontinência urinária foi realizada com populações brancas, e alguns dados comparativos sugerem que mulheres caucasianas têm maior suscetibilidade de desenvolver incontinência urinária quando comparadas a mulheres afrodescendentes 23 . Kubik et al. 22 observaram que as mulheres caucasianas tinham maior conhecimento sobre a incontinência urinária e sobre como lidar com esse problema de saúde; no entanto, a etnia deixou de ter associação com o conhecimento sobre a incontinência urinária quando ajustado pelo status socioeconômico. Por outro lado, em nosso estudo, mesmo na análise ajustada, houve associação entre etnia não caucasiana e maior prevalência de incontinência urinária. $\mathrm{O}$ fato de termos encontrado maior prevalência de incontinência urinária em mulheres não caucasianas, o que diverge da literatura, talvez possa ser explicado pelo nível de miscigenação das mulheres participantes desta investigação, se comparado ao de outros países.

Nesta pesquisa, a prevalência de incontinência urinária referida entre as mulheres com idade entre 45 e 55 anos foi equivalente a 1,35 vez a prevalência observada entre as mulheres com idade inferior a 45 anos, ao passo que a prevalência de incontinência urinária referida entre as mulheres com idade superior a 55 anos foi equivalente a 1,88 vez a prevalência observada entre as mulheres com idade inferior a 45 anos. Esse resultado vai ao encontro de outras pesquisas que apresentam a idade como um dos principais fatores associados à incontinência urinária 5,7. As alterações hormonais que surgem com o avanço da idade, sobretudo na pós-menopausa, desencadeiam uma queda do nível de estrogênio que está presente na bexiga, na uretra, na vagina e na musculatura do assoalho pélvico, comprometendo a função do trato urinário inferior 24 .

Nosso estudo teve como diferencial incluir mulheres mais jovens (idade inferior a 45 anos) e demonstrou alta prevalência de autorrelato de incontinência urinária nessas participantes (23,9\%). Esse achado é indicativo de que os sintomas surgem cedo e, desse modo, é possível a atuação preventiva precoce.

Foi possível verificar, ainda, que a prevalência de incontinência urinária nas mulheres com escolaridade baixa (Ensino Fundamental completo/incompleto) equivale a 1,59 vez a prevalência observada entre as mulheres com escolaridade alta. Waetjen et al. 7 encontraram que a baixa escolaridade esteve associada à incontinência urinária de urgência. O baixo nível de escolaridade pode estar relacionado à falta de procura por serviço médico entre mulheres com sintomas de incontinência urinária devido à carência de informações e ao fato de elas julgarem que a incontinência urinária faz parte de um processo natural de envelhecimento 10. Por isso, é necessário haver maior preparo dos profissionais da saúde para abordar esses temas, bem como a disponibilização de alternativas terapêuticas para essa afecção por parte do Sistema Único de Saúde.

Dentre os fatores comportamentais investigados, apenas a autoavaliação do estado de saúde manteve-se associada à incontinência 
urinária referida após análise hierarquizada. A prevalência de incontinência urinária entre as mulheres com pior estado de saúde foi $1,35 \mathrm{vez}$ a prevalência das mulheres com melhor estado de saúde. De acordo com estudos sobre a qualidade de vida, a percepção do estado de saúde é pior em mulheres com incontinência urinária, sendo necessário compreender os efeitos dos sintomas e melhorar as estratégias de intervenção 25,26.

$\mathrm{O}$ excesso de peso corporal esteve associado à incontinência urinária referida somente na análise bruta, pois sofreu confundimento pelas variáveis sociodemográficas. Segundo diversas pesquisas 4,5,9,23,27, o sobrepeso predispõe à incontinência urinária, no entanto há poucos dados consistentes sobre os mecanismos biológicos que agem sobre esse efeito dose-resposta 23. É provável que o aumento da pressão intra-abdominal gerado pelo excesso de peso interfira no mecanismo de controle vesical 5 . Todavia, ainda falta identificar se a incontinência pode ser um fator contribuinte para o desenvolvimento da obesidade, já que algumas mulheres deixam de praticar atividade física em função dos sintomas, que podem agravar-se em atividades de alto impacto e que elevam a pressão intra-abdominal.

A prática de atividade física não obteve associação significativa com a incontinência urinária, dado que corrobora o estudo de Moller et al. 27, realizado na Dinamarca; outras pesquisas, porém, mostram que a prática de atividade física está associada a uma menor probabilidade de a mulher apresentar incontinência urinária 9,28. Por outro lado, sabe-se, também, que a atividade física de alto impacto exerce aumento da pressão intra-abdominal, intensificando os sintomas de perda urinária, especialmente em atletas de ginástica e atletismo 29,30. Assim, os resultados das pesquisas em relação à prática de atividade física ainda se mostram controversos. Nosso estudo apresentou limitações na quantificação do nível de atividade física, já que o autorrelato dificulta a obtenção de resultados mais precisos.

Houve associação entre constipação e incontinência urinária somente na análise bruta, embora já se tenha mencionado que a constipação crônica pode favorecer o surgimento da afecção em estudo 14,27. Está claro que a incontinência urinária é uma condição dinâmica, podendo ocorrer variações de seus sintomas ao longo do tempo. A constipação é um aspecto passível de ser alterado com mudanças de comportamento, como a adoção de hábitos alimentares saudáveis e a prática de atividade física 14.

Considerando as limitações do presente estudo, sugere-se que futuras pesquisas utilizem inquéritos domiciliares populacionais e, preferencialmente, estudos de coorte longitudinais para verificar a relação de causalidade entre as variáveis. Propõe-se, além disso, que sejam utilizados questionários validados para avaliação da incontinência urinária, como o ICIQ-SF, e avaliados os fatores obstétricos (via de parto, número de partos), hormonais (menopausa) e referentes a doenças associadas.

O presente estudo foi relevante por abranger os fatores sociodemográficos e comportamentais associados à incontinência urinária em um grupo de mulheres que procuram um serviço de saúde de referência para exame de rastreamento do câncer de colo uterino em Florianópolis. A elevada prevalência de incontinência urinária nesse grupo sugere que esse serviço de atenção à saúde da mulher possa ser expandido para o controle de outras condições de saúde que afetam a qualidade de vida. Além de investigar os fatores associados à incontinência urinária, poderiam ser utilizadas medidas de prevenção primária e secundária direcionadas à incontinência urinária na população geral feminina. 


\section{Resumen}

Este estudio transversal tuvo como objetivo investigar la asociación entre factores socio-demográficos y de comportamiento con la presencia de incontinencia urinaria, informada por 784 mujeres que solicitaron un examen preventivo de cáncer de cuello uterino en el área metropolitana de Florianópolis, Santa Catarina, Brasil. Se obtuvieron datos socio-demográficos, estado de salud, actividad física, estreñimiento e índice de masa corporal, utilizándose el International Consultation on Incontinence Questionnaire-SF. La prevalencia de incontinencia urinaria fue de un 30,7\% (16,5\% perdían orina una vez por semana o menos y un $23,8 \%$ en pequeña cantidad). En la regresión de Poisson bruta se asoció a la incontinencia urinaria: la baja escolaridad; renta por persona de la familia de hasta un salario mí nimo; etnia no caucásica; exceso de peso corporal; peor autoevaluación de estado de salud; estreñimiento y edad. Tras los ajustes, siguiendo un modelo jerarquizado, permanecieron asociados: escolaridad; etnia; estado de salud y edad. La alta prevalencia de incontinencia urinaria de mujeres que solicitaron un examen de indicios de cáncer de cuello uterino justifica aproximaciones preventivas en esos espacios.

Incontinencia Urinaria; Salud de la Mujer; Factores de Riesgo; Tamizaje Masivo; Actividad Motora

\section{Colaboradores}

C. Sacomori participou da redação do projeto, coleta organização e análise dos dados e escrita do artigo. N. B. Negri participou da redação do projeto, coleta e organização dos dados e redação do artigo. F. L. Cardoso participou da redação do projeto, análise dos dados e redação do artigo.

\section{Agradecimentos}

Agradecemos ao grupo de mulheres voluntárias da Rede Feminina de Combate ao Câncer de Florianópolis, pela oportunidade de realizarmos este estudo.

\section{Referências}

1. Abrams P, Cardozo L, Fall M, Griffiths D, Rosier P, Ulmsten $\mathrm{U}$, et al. The standardization of terminology of lower urinary tract function: report from the standardisation sub-committee of the International Continence Society. Neurourol Urodyn 2003; 61:37-49.

2. Rios AAN, Cardoso JR, Rodrigues MA, de Almeida SH. The help-seeking by women with urinary incontinence in Brazil. Int Urogynecol J 2011, 22:879-84.

3. Jokhio A, Rizvi R, Rizvi J, Macarthur C. Urinary incontinence in women in rural Pakistan: prevalence, severity, associated factors and impact on life. BJOG 2013; 120:180-6.

4. Hannestad YS, Rortveit G, Sandvik H, Hunskaar S. A community-based epidemiological survey of female urinary incontinence: the Norwegian Epicont study. J Clin Epidemiol 2000; 53:1150-7.
5. Fornell EU, Wingren G, Kjolhede P. Factors associated with pelvic floor dysfunction with emphasis on urinary and fecal incontinence and genital prolapse: an epidemiological study. Acta Obstet Gynecol Scand 2004; 83:383-9.

6. Ojengbede OA, Morhason-Bello IO, Adedokun BO, Okonkwo NS, Kolade CO. Prevalence and the associated trigger factors of urinary incontinence among 5000 black women in sub-Saharan Africa: findings from a community survey. BJU Int 2011; 107:1793-800.

7. Waetjen LE, Liao S, Johnson WO, Sampselle CM, Sternfield B, Harlow SD, et al. Factors associated with prevalent and incident urinary incontinence in a cohort of midlife women: a longitudinal analysis of data. Am J Epidemiol 2007; 165:309-18. 
8. Timur-Taşhan S, Beji NK, Aslan E, Yalçin Ö. Determining lower urinary tract symptoms and associated risk factors in young women. Int J Gynecol Obst 2012; 118:27-30.

9. Qiu J, Lv L, Lin X, Long L, Zhu D, Xu R, et al. Body mass index, recreational physical activity and female urinary incontinence in Gansu, China. Eur J Obstet Gynecol Reprod Biol 2011; 159:224-9.

10. Guarisi T, Pinto Neto AM, Osis MJ, Pedro AO, Costa Paiva LH, Faúndes A. Incontinência urinária entre mulheres climatéricas brasileiras: inquérito domiciliar. Rev Saúde Pública 2001; 35:428-35.

11. Oliveira E, Zuliani LMM, Ishicava J, Silva SV, Albuquerque SSR, Souza AMB, et al. Avaliação dos fatores relacionados à ocorrência da incontinência urinária feminina. Rev Assoc Med Bras (1992) 2010; 56:688-90.

12. Albuquerque KM, Frias PG, Andrade CLT, Aquino EML, Menezes G, Szwarcwald CL. Cobertura do teste de Papanicolaou e fatores associados à nãorealização: um olhar sobre o Programa de Prevenção do Câncer do Colo do Útero em Pernambuco, Brasil. Cad Saúde Pública 2009; 25 Suppl 2:S301-9.

13. Gasperin SI, Boing AF, Kupek E. Cobertura e fatores associados à realização do exame de detecção do câncer de colo de útero em área urbana no Sul do Brasil: estudo de base populacional. Cad Saúde Pública 2011; 27:1312-22.

14. Amselem C. Constipation: a potential cause of pelvic floor damage? Neurogastroenterol Motil 2010; 22:150-e48.

15. Tamanini JTN, Dambros M, D'Ancona CAL, Rodrigues-Palma PC, Netto Jr NR. Validação para o português do "International Consultation on Incontinence Questionnaire - Short Form” (ISIQ-SF). Rev Saúde Pública 2004; 38:438-44.

16. Mascarenhas CHM, Prado FO, Fernandes MH. Fatores associados à qualidade de vida de agentes comunitários de saúde. Ciênc Saúde Coletiva; no prelo.

17. Hosmer DW, Lemeshow S. Applied logistic regression. New York: Wiley; 1989.

18. Pedro AO, Pinto Neto AM, Paiva LHCS, Osis MJ, Hardy E. Idade de ocorrência da menopausa natural em mulheres brasileiras: resultados de um inquérito populacional domiciliar. Cad Saúde Pública $2003 ; 19: 17-25$
19. Geelen JM, Hunskaar S. The epidemiology of female urinary incontinence. European Clinics in Obstetrics and Gynaecology 2005; 1:3-11.

20. De Araujo MP, Takano CC, Girão MJBC, Sartori MGF. Pelvic floor disorders among indigenous women living in Xingu Indian Park, Brazil. Int Urogynecol J 2009; 20:1079-84.

21. Fiegen MM, Benson KD, Hanson JD, Prasek J, Hansen K, VanEerden P. The prevalence of urinary incontinence in American Indian women from a South Dakota tribe. Int Urogynecol J 2012; 23:473-9.

22. Kubik K, Blackwell L, Heit M. Does socioeconomic status explain racial differences in urinary incontinence knowledge? Am J Obstet Gynecol 2004; 191:188-93.

23. Hunskaar S. A systematic review of overweight and obesity as risk factors and targets for clinical intervention for urinary incontinence in women. Neurourol Urodyn 2008; 27:749-57.

24. Robinson D, Cardozo L. Estrogens and the lowe urinary tract. Neurourol Urodyn 2011; 30:754-7.

25. Ko Y, Lin SJ, Salmon JW, Bron MS. The impact of urinary incontinence on quality of life of the elderly. Am J Manag Care 2005; 11(4 Suppl):103-11.

26. Dedicação AC, Haddad M, Saldanha MES, Driusso P. Comparação da qualidade de vida nos diferentes tipos de incontinência urinária feminina. Rev Bras Fisioter 2009; 13:116-22.

27. Moller LA, Lose G, Jorgensen T. Risk factors for lower urinary tract symptoms in women 40 to 60 years of age. Obstet Gynecol 2000; 93:446-51.

28. Danforth KN, Shah AD, Townsend MK, Lifford KL, Curhan GC, Resnick NM, et al. Physical activity and urinary incontinence among healthy, older women. Am J Obstet Gynecol 2007; 109:721-7.

29. Bo K. Urinary incontinence, pelvic floor dysfunction, exercise and sport. J Sports Med 2004; 34:451-64.

30. Guerra MC. La incontinencia urinaria en la mujer deportista de élite. Rev Iberoam Fisioter Kinesiol 2006; 9:78-89.

Recebido em 07/Set/2012

Versão final reapresentada em 28/Jan/2013

Aprovado em 06/Fev/2013 